\title{
Quality Assessment of Radix Salviae Miltiorrhizae
}

\author{
Ping Hu, ${ }^{a, b}$ Guo-An LuO, ${ }^{b, c}$ Zhongzhen ZHAO, ${ }^{a}$ and Zhi-Hong JiAnG ${ }^{*, a}$ \\ ${ }^{a}$ School of Chinese Medicine, Hong Kong Baptist University; Kowloon Tong, Hong Kong: ${ }^{b}$ School of Chemistry and \\ Pharmaceutics, East China University of Science and Technology; Shanghai 200237, China: and ${ }^{c}$ Analysis Center, \\ Tsinghua University; Beijing 100084, China. Received October 25, 2004; accepted December 15, 2004
}

This paper describes an improved quality assessment method for Radix Salviae Miltiorrhizae (Root of Salvia miltiorrhiza BGE.) which was established using chromatographic fingerprinting and quantification of multiple marker compounds in the crude drug. High-performance thin-layer chromatography (HPTLC) fingerprinting of water-soluble phenolics and nonpolar tanshinones was performed separately and the authentication of Radix Salviae Miltiorrhizae was achieved by comparing the fingerprints of the samples with those of the reference crude drug and by comparing the $R f$ values of the bands in TLC fingerprints with those of reference compounds. HPLC fingerprints were obtained by simultaneous separation of phenolics and diterpenoids in Radix Salviae Miltiorrhizae. The HPLC fingerprints of seven batches of samples from different regions of China showed similar chromatographic patterns, and seven peaks were selected as characteristic peaks. The relative retention time of these characteristic peaks in the HPLC fingerprints was established as an important parameter for the identification of this herbal medicine. The pharmacologically active marker compounds salvianolic acid $B$, rosmarinic acid, and tanshinone IIA in herbal medicine were quantitatively determined using reverse-phase HPLC techniques. The HPLC quantitation methods of the three marker compounds were validated and the measurement uncertainty, which is important for setting the proposed content limit of the marker compounds in herbal medicine, were further evaluated.

Key words Salvia miltiorrhiza; quality assessment; HPLC; chromatographic fingerprint; phenolics; diterpenoids

Radix Salviae Miltiorrhizae (danshen, 丹参), the dried root of Salvia miltiorrhiza BGE. (Family Labiatae), is a very important traditional Chinese medicine that promotes blood flow to overcome blood stasis and to cool the blood to resolve abscesses. ${ }^{1)}$ Modern pharmacological and clinical studies showed that Radix Salviae Miltiorrhizae has curative effects in cardiovascular disease. ${ }^{2)}$ In China, Radix Salviae Miltiorrhizae is widely used in many preparations of Chinese medicines. The consumption of this crude drug was evaluated at about 80 million kilogram a year in China.

The chemical constituents of Radix Salviae Miltiorrhizae have been thoroughly studied for more than 60 years. The research results indicated that it contains a variety of diterpenoids, phenolics, flavonoids, triterpenoids, and sterols. ${ }^{3)}$ Pharmacological studies showed that nonpolar compounds such as tanshinone IIA, tanshinone I, and cryptotanshinone are active components with a variety of biological activities including antiischemic, antioxidant, and antitumor properties. ${ }^{4,5)}$ In recent years, the phenolics have attracted more attention since they are the main components in the water decoction that is the most common dosage form administered to patients in Chinese clinics. In the water extract, apart from a few simple benzoic derivatives, caffeic acid metabolites are the major compounds. ${ }^{6,7)}$ These caffeic metabolites include caffeic acid monomer (such as caffeic acid itself and danshensu), dimers (such as rosmarinic acid), trimers (such as lithospermic acid), and tetramers (such as salvianolic acid B). These phenolics were shown to protect the myocardium from ischemia-induced derangement, protect neural cells from injuries caused by anoxia, inhibit platelet aggregation, reduce hepatic fibrosis, and inhibit the activities of HIV-1. ${ }^{8,9)}$

Since Radix Salviae Miltiorrhizae and its medicinal products are so commonly used clinically, a reasonable assessment method for evaluating the quality of the crude drug is essential to ensure the efficiency and stability of its products.
On the other hand, while several bases of Good Agriculture Practice (GAP) have been built to grow the medicinal plant Salvia miltiorrhiza in China, it is also very important to establish reasonable assessment methods for the quality control of plants and raw materials.

A chromatographic fingerprint is in practice a chromatographic pattern of common types of chemical components in complicated samples. ${ }^{10}$ The HPLC fingerprint is a powerful tool for the identification of traditional Chinese medicines due to the high separation ability of HPLC. In the U.S.A. and Europe, HPLC chromatographic fingerprinting was used to establish official standards for the quality control of herbal dietary supplements or herbal medicine products. In China, guidelines for the establishment of fingerprints for injections of Chinese medicines have been officially published. ${ }^{11)}$ It is generally recognized that both water-soluble compounds and nonpolar compounds in Chinese herbal medicines can be simultaneously separated on HPLC. Thus more information helpful in distinguishing false herbs and evaluating the quality of crude drugs can be obtained when chromatography is coupled with spectrometric analysis, such as diode array detector (DAD) and mass spectrometry. ${ }^{12-14)}$ Therefore, if the HPLC fingerprint technique is used in the quality standard for the identification of herbal medicines, the reliability of identification results will improve markedly.

In addition to the identification of crude drugs, quantitative determination of marker compounds is another useful method to control the quality of Chinese medicines. In the Chinese Pharmacopoeia, the quantification of Radix Salviae Miltiorrhizae is performed by determination of tanshinone IIA with HPLC. Although tanshinone IIA is an important ingredient in crude drugs, its content is not sufficient for comprehensively evaluating the quality of the herbal medicine because tanshinone $\mathrm{II}_{\mathrm{A}}$ is not the sole pharmaceutically active compound. Since there are water-soluble constituents 
(salvianolic acid B, etc.) and nonpolar compounds (tanshinone IIA, etc.) in Radix Salviae Miltiorrhizae, a quality assessment method dealing with both of these constituents should be established to evaluate the quality of the crude drug comprehensively and effectively.

In this study, an improved quality assessment method for Radix Salviae Miltiorrhizae was established using chromatographic fingerprints and HPLC determination of the pharmacologically active constituents in the crude drug. HPTLC fingerprinting of water-soluble phenolics and nonpolar tanshinones were studied, and the multicomponent HPLC fingerprint method was established with simultaneous separation of phenolics and tanshinones in Radix Salviae Miltiorrhizae. LC-MS-MS techniques were employed to identify the major constituents in the HPLC fingerprint chromatogram. For the application of this HPLC fingerprinting technique, seven batches of Radix Salviae Miltiorrhizae samples collected from different regions of China and three batches of processed samples purchased from Hong Kong were analyzed. In addition, three pharmacologically active components, salvianolic acid $\mathrm{B}$, rosmarinic acid, and tanshinone IIA, in herbal medicine were quantitatively determined using reverse-phase HPLC techniques. The HPLC quantitation methods of three marker compounds were validated, and the measurement uncertainty (MU), which is important for setting the proposed content limit of marker compounds in herbal medicine, were further evaluated.

\section{Results and Discussion}

HPTLC Fingerprinting Identification The performance of TLC using a sorbent with a homogeneous particle size of about $5 \mu \mathrm{m}$ and with narrow particle size distribution has been confirmed to be superior to that of the usual TLC plate that has long been used in almost all pharmacopeias for the identification of herbal raw materials. ${ }^{15)}$ Therefore in the study of quality assessment of Radix Salviae Miltiorrhizae, HPTLC plates were used to establish the TLC fingerprint method. The chromatographic conditions, especially the developing solvents (type of solvents and ratio), were carefully optimized before the seven batches of crude drug samples were analyzed. The results observed under UV light and visible light showed a good separation for both nonpolar compounds (Fig. 1) and water-soluble compounds (Fig. 2).

Reference Chinese Materia Medica and reference marker compounds of tanshinone IIA, cryptotanshinone (for the nonpolar group), rosmarinic acid, and salvianolic acid B (for the water-soluble group) were separated on the same plates for the authentication of unknown samples. The samples were identified by comparing their fingerprints with those of reference Chinese Materia Medica compounds and comparing the $R f$ values of bands in their fingerprints with those of marker compounds. For positive identification, the sample should show bands with chromatographic characteristics, including color and position, similar to those of reference Chinese Materia Medica compounds. The sample should show bands with the same color and $R f$ values ( $c a$. 0.59 for tanshinone IIA, $c a$. 0.34 for cryptotanshinone, $c a$. 0.43 for rosmarinic acid, ca. 0.20 for salvianolic acid B), similar to those of the reference marker compounds.

HPLC Fingerprinting Identification HPLC fingerprinting for simultaneous separation of both phenolics and
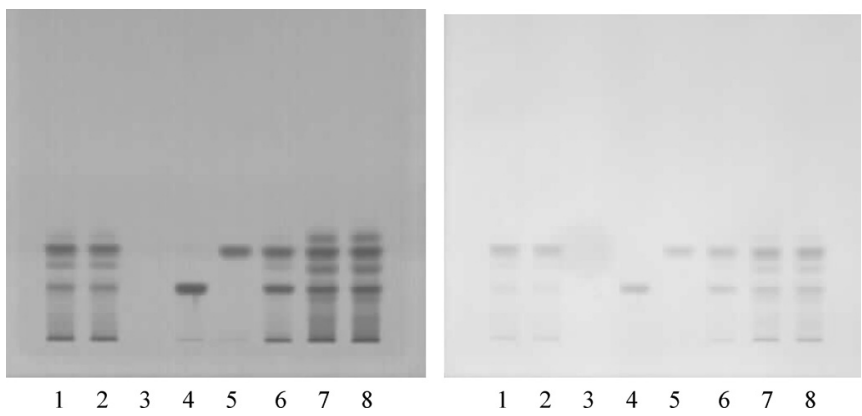

Fig. 1. HPTLC Fingerprints of Tanshinones in Radix Salviae Miltiorrhizae

Left, observed under UV $254 \mathrm{~nm}$; right, observed under visible light; $R f$ value for cryptotanshinone and tanshinone IIA is 0.34 and 0.59 , respectively. 1 , Sample; 2 , sample duplicate; 3 , solvent blank; 4, cryptotanshinone; 5 , tanshinone IIA; 6 , spiked sample; 7 , reference drug; 8 , reference drug duplicate.
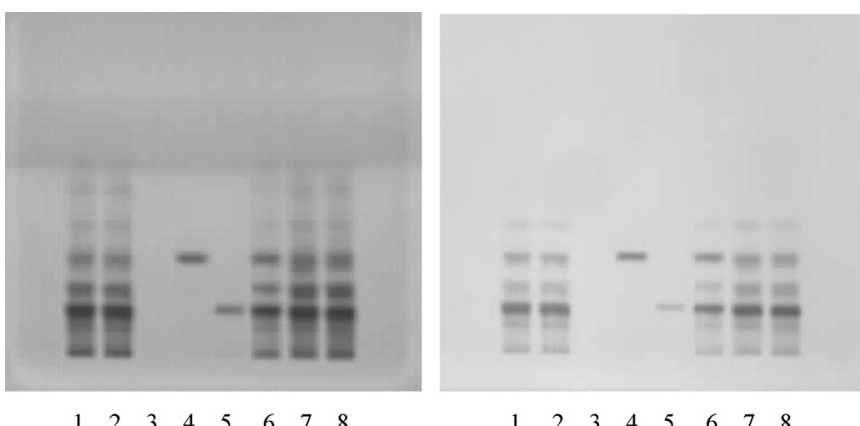

$\begin{array}{llllllll}1 & 2 & 3 & 4 & 5 & 6 & 7 & 8\end{array}$

Fig. 2. HPTLC Fingerprints of Phenolic Compounds in Radix Salviae Miltiorrhizae

Left, observed under UV $254 \mathrm{~nm}$; right, observed under visible light after spraying with $2 \% \mathrm{FeCl}_{3} / \mathrm{EtOH} ; R f$ value for rosmarinic acid and salvianolic acid $\mathrm{B}$ is 0.43 and 0.20 , respectively. 1 , Sample; 2 , sample duplicate; 3 , solvent blank; 4 , rosmarinic acid; 5 , salvianolic acid B; 6 , spiked sample; 7 , reference drug; 8 , reference drug duplicate.

tanshinones in the herbal medicine were studied. First, the extracting procedure of crude drug was investigated. Because the polarities between phenolics and tanshinones are different, a two-step extracting procedure was used to extract the tanshinones with methanol, followed by extracting the phenolics with boiling water. Then the HPLC separation conditions such as mobile phase, gradient program, and detection wavelength were optimized to carry out simultaneous separation of two groups of compounds with good resolution. Finally, several peaks in the HPLC fingerprint profile were structurally identified employing LC-MS-MS techniques and comparing the retention time values of reference standards with those of the peaks in the fingerprint chromatogram. Figure 3 shows the typical fingerprint chromatograms of Radix Salviae Miltiorrhizae and the results of LC-MS-MS identification.

The HPLC fingerprints of seven batches of samples from different regions of China were obtained under optimized conditions showed similar chromatographic patterns. After carefully analyzing the fingerprint profiles of these samples, seven peaks with reasonable heights and good resolution were selected as characteristic peaks for the identification of the crude drug because these peaks represent major active compounds and were chemically identified by LC-MS-MS analysis. ${ }^{16)}$ They were danshensu (peak 1), rosmarinic acid (peak 8), lithospermic acid (peak 9), salvianolic acid B (peak 


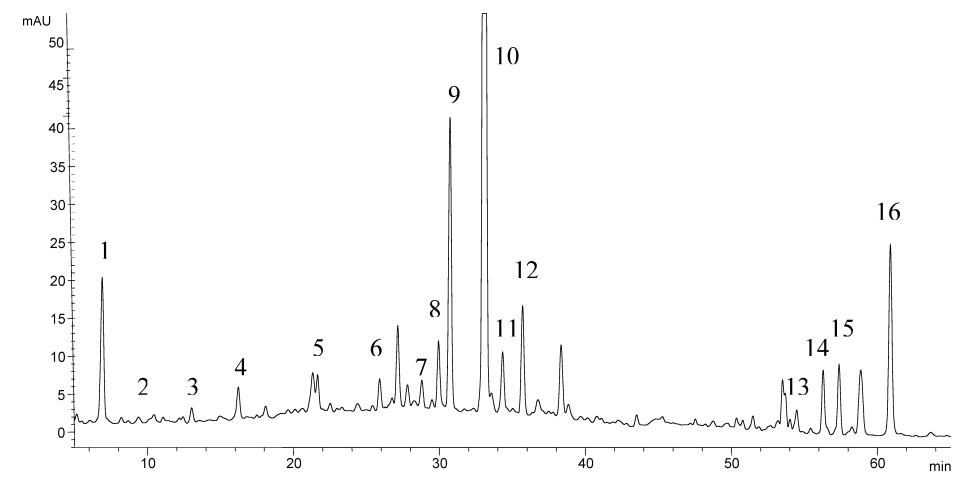

Fig. 3. Representative Fingerprinting Chromatogram of Radix Salviae Miltiorrhizae

1, Danshensu; 2, protocatechuic acid; 3, protocatechualdehyde; 4, caffeic acid; 5, salvianolic acid F; 6, salvianolic acid D; 7, salvianolic acid E; 8, rosmarinic acid; 9, lithospermic acid; 10, salvianolic acid B; 11, isomer of salvianolic acid B; 12, salvianolic acid A; 13, dihydrotanshinone I; 14, cryptotanshinone; 15, tanshinone I; 16, tanshinone IIA.

Table 1. Relative Retention Time (RRT) of 7 Characteristic Peaks in HPLC Fingerprints of 7 Batches of Radix Salviae Miltiorrhizae

\begin{tabular}{|c|c|c|c|c|c|c|c|}
\hline $\begin{array}{l}\text { Batch } \\
\text { No. }\end{array}$ & $\begin{array}{c}\text { Peak } 1 \\
\text { (Danshensu) }\end{array}$ & $\begin{array}{c}\text { Peak } 8 \\
\text { (Rosmarinic acid) }\end{array}$ & $\begin{array}{c}\text { Peak } 9 \\
\text { (Lithospermic acid) }\end{array}$ & $\begin{array}{c}\text { Peak } 10 \\
\text { (Salvianolic acid B) }\end{array}$ & $\begin{array}{c}\text { Peak 11 } \\
\text { (Isomer of } \\
\text { salvianolic acid B) }\end{array}$ & $\begin{array}{c}\text { Peak } 14 \\
\text { (Cryptotanshinone) }\end{array}$ & $\begin{array}{c}\text { Peak } 16 \\
\text { (Tanshinone } \\
\text { IIA) }\end{array}$ \\
\hline 1 & $0.23^{a)}\left(0.21^{b)}\right)$ & $1.00(0.91)$ & $1.03(0.93)$ & $1.10(1.00)$ & $1.15(1.04)$ & $1.88(1.71)$ & $2.04(1.85)$ \\
\hline 2 & $0.23(0.21)$ & $1.00(0.90)$ & $1.03(0.92)$ & $1.11(1.00)$ & $1.15(1.04)$ & $1.90(1.72)$ & $2.05(1.85)$ \\
\hline 3 & $0.23(0.21)$ & $1.00(0.90)$ & $1.03(0.92)$ & $1.11(1.00)$ & $1.15(1.04)$ & $1.89(1.71)$ & $2.04(1.84)$ \\
\hline 4 & $0.24(0.21)$ & $1.00(0.90)$ & $1.03(0.93)$ & $1.11(1.00)$ & $1.15(1.04)$ & $1.90(1.72)$ & $2.04(1.84)$ \\
\hline 5 & $0.23(0.21)$ & $1.00(0.91)$ & $1.03(0.93)$ & $1.10(1.00)$ & $1.15(1.04)$ & $1.88(1.70)$ & $2.03(1.84)$ \\
\hline 6 & $0.23(0.21)$ & $1.00(0.91)$ & $1.02(0.93)$ & $1.10(1.00)$ & $1.14(1.04)$ & $1.87(1.70)$ & $2.03(1.84)$ \\
\hline 7 & $0.23(0.21)$ & $1.00(0.91)$ & $1.03(0.93)$ & $1.10(1.00)$ & $1.15(1.04)$ & $1.89(1.71)$ & $2.03(1.84)$ \\
\hline Mean & $0.23(0.21)$ & $1.00(0.91)$ & $1.03(0.93)$ & $1.10(1.00)$ & $1.15(1.04)$ & $1.89(1.71)$ & $2.04(1.84)$ \\
\hline S.D. & $0.004(0.002)$ & $-(0.001)$ & $0.004(0.001)$ & $0.005(-)$ & $0.004(0.001)$ & $0.01(0.009)$ & $0.008(0.005)$ \\
\hline RSD (\%) & $1.6(0.68)$ & $-(0.17)$ & $0.37(0.06)$ & $0.48(-)$ & $0.33(0.08)$ & $0.59(0.55)$ & $0.37(0.27)$ \\
\hline
\end{tabular}

a) RRT calculated by setting peak of romarinic acid as marker peak. b) RRT calculated by setting peak of salvianolic acid B as marker peak.

10), salvianolic acid B isomer (peak 11), cryptotanshinone (peak 14), and tanshinone IIA (peak 16). Relative retention times (RRT) of seven characteristic peaks were calculated using the following equation.

$\mathrm{RRT}=$ retention time of characteristic peak/retention time of marker peak

The calculated results of seven batches of samples (Table 1) indicated that the RRTs of the seven characteristic peaks were sufficiently stable to be set as the parameter for identification when the peak of rosmarinic acid or salvianolic acid B was chosen as the marker peak. Thus the sample with a similar HPLC chromatographic pattern and RRT value compared with the typical fingerprint chromatogram and average RRT shown in Fig. 3 and Table 1, respectively, can be authenticated as genuine Radix Salviae Miltiorrhizae.

Validation Results of Quantitative Analysis HPLC chromatograms of the quantification of tanshinone IIA, rosmarinic acid, and salvianolic acid B in Radix Salviae Miltiorrhizae are shown in Figs. 4 and 5, respectively. After carefully optimizing the HPLC conditions including the mobile phase and detection wavelength, the method validation for assay of three pharmacologically active marker compounds was performed. The validation results (calibration curve, precision, repeatability, recovery, detection limits, quantification limits) are listed in Table 2. Good linearity, precision of injection, and repeatability $(\mathrm{RSD}<2 \%$ ) were demonstrated for the three compounds. The limit of quantitation (LOQ) is much lower than the content of the three pharmacologically active marker compounds in the crude drug. This indicates

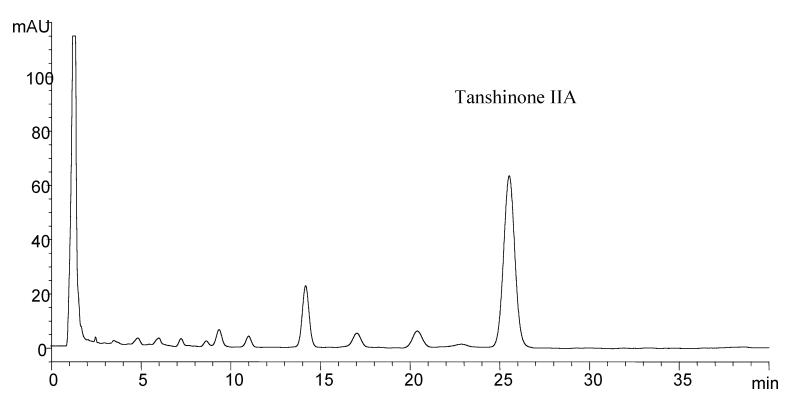

Fig. 4. HPLC Chromatogram for the Quantitation of Tanshinone IIA

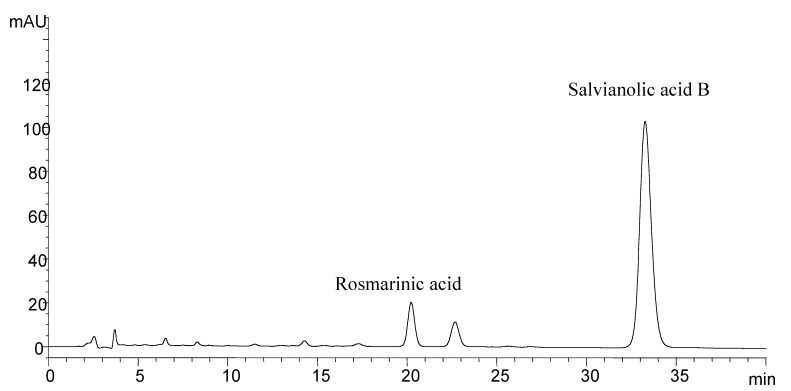

Fig. 5. HPLC Chromatogram for the Quantitation of Rosmarinic Acid and Salvianolic Acid B

that the established quantification method is suitable for the quantitative analysis of Radix Salviae Miltiorrhizae.

Measurement Uncertainty MU is an important parameter in analysis results. It is also useful to set the proposed 
Table 2. Validation Data for HPLC Quantitative Method of Radix Salviae Miltiorrhizae

\begin{tabular}{|c|c|c|c|c|c|c|c|}
\hline Components & $\begin{array}{c}\text { Calibration } \\
\text { equation }\end{array}$ & $\begin{array}{l}\text { Correlation } \\
\text { coefficient }\end{array}$ & $\begin{array}{l}\text { Precision } \\
\text { RSD (\%) }\end{array}$ & $\begin{array}{c}\text { Repeatability } \\
\text { RSD (\%) }\end{array}$ & $\begin{array}{c}\text { Recovery } \\
(\%)\end{array}$ & $\begin{array}{l}\text { MDL } \\
(\mu \mathrm{g} / \mathrm{g})\end{array}$ & $\begin{array}{l}\mathrm{LOQ} \\
(\mu \mathrm{g} / \mathrm{g})\end{array}$ \\
\hline Tanshinone IIA & $y=107.788 x-5.481$ & 1.0000 & 0.12 & 1.4 & 100.4 & 1.7 & 10 \\
\hline Rosmarinic acid & $y=61.647 x-0.618$ & 1.0000 & 0.75 & 1.3 & 97.9 & 14 & 100 \\
\hline Salvianolic acid B & $y=18.279 x-3.317$ & 1.0000 & 0.29 & 1.2 & 97.0 & 70 & 500 \\
\hline
\end{tabular}

limits of the contents of marker compounds in Chinese medicine. In our study, the estimation of MU using the method validation data was based on the guides of Eurachem and Valid Analytical Measurement. ${ }^{17,18)}$ The most significant sources of MU, including both random effects (precision study and sample homogeneity) and systematic effects (bias study and standard purity), which contribute to the overall uncertainty were considered during the uncertainties evaluation (Table 3).

Precision (Repeatability): The results obtained from analyses of seven batches of representative Radix Salviae Miltiorrhizae samples were statistically analyzed. Each batch of samples was determined in duplicate in separate extraction and HPLC runs. The absolute deviation from the means for duplicate tests of different samples were pooled together to give an estimate of relative standard deviation $u(P) / P$.

Bias (Recovery): The bias of the analytical procedure was investigated during the method validation study using spiked samples. The bias of the analytical procedure was estimated using the recovery data of Chinese herbal medicine samples fortified with reference standards of tanshinone IIA, rosmarinic acid, and salvianolic acid B. The relative standard uncertainty $u(\overline{\operatorname{Re} c}) / \overline{\operatorname{Re} c}$ was calculated as the relative standard deviation of the mean value. In addition, Student's $t$-test was carried out to determine whether the mean recovery was significantly different from 1 . If the recovery is not significantly different from 1 , the uncertainty does not need to be increased to take into account that the recovery has not been corrected for.

Standard Purity: The purity of reference standards was provided by the manufacturer. The standard uncertainty $u\left(c_{\text {std }}\right) / c_{\text {std }}$ in purity was calculated using the assumption of a rectangular distribution.

Sample Homogeneity: To investigate uncertainty derived from sample homogeneity, herbal medicines were sampled using the quartering process prior to the grinding step, which was usually used to ensure the best sample homogeneity. The result revealed that the relative standard uncertainty $u(F($ hom $) / F($ hom $))$ derived from sample homogeneity is the major factor contributing to the overall MU.

Combined Standard Uuncertainty: During the study of the analytical procedure, uncertainty sources, including the precision, bias, standard purity, and sample homogeneity were thoroughly investigated. These four relative standard uncertainties were combined using the square root of the summed variances to give the overall $\mathrm{MU}\left[u_{\mathrm{c}}(c)\right]$.

Expanded Uncertainty: The expanded uncertainty $u(c)$ at the $95 \%$ confidence level for the determination of marker compounds in Radix Salviae Miltiorrhizae was calculated by multiplying the combined standard uncertainty with a coverage factor of 2. Thus, low- or high-level results would be reported with the uncertainties expressed in concentration
Table 3. Measurement Uncertainties for the HPLC Determination of Marker Compounds in Radix Salviae Miltiorrhizae

\begin{tabular}{cccc}
\hline \hline $\begin{array}{c}\text { Standard } \\
\text { uncertainties }\end{array}$ & $\begin{array}{c}\text { Tanshinone } \\
\text { IIA }\end{array}$ & $\begin{array}{c}\text { Rosmarinic } \\
\text { acid }\end{array}$ & $\begin{array}{c}\text { Salvianolic } \\
\text { acid B }\end{array}$ \\
\hline$u(P) / P$ & 0.008 & 0.011 & 0.009 \\
$u(\operatorname{Re} c) / \overline{\operatorname{Re} c}$ & 0.0024 & 0.0093 & 0.012 \\
$u\left(c_{\text {std }}\right) / c_{\text {std }}$ & 0.0058 & 0.0087 & 0.0058 \\
$u(F($ hom $) / F($ hom $))$ & 0.069 & 0.071 & 0.066 \\
$u_{\mathrm{c}}(c)$ & $0.070 \mathrm{c}$ & $0.073 \mathrm{c}$ & $0.068 \mathrm{c}$ \\
$U(c)$ & $0.14 \mathrm{c}$ & $0.15 \mathrm{c}$ & $0.14 \mathrm{c}$ \\
\hline
\end{tabular}

Table 4. Contents of Three Marker Compounds in Crude Drugs of Radix Salviae Miltiorrhizae

\begin{tabular}{cccc}
\hline \hline Source & $\begin{array}{c}\text { Tanshinone } \\
\text { IIA (\%) }\end{array}$ & $\begin{array}{c}\text { Rosmarinic } \\
\text { acid (\%) }\end{array}$ & $\begin{array}{c}\text { Salvianolic } \\
\text { acid B (\%) }\end{array}$ \\
\hline $\begin{array}{c}\text { Sichuan Province, China } \\
\text { Yi Nan, Shandong Province, China }\end{array}$ & 0.082 & 0.19 & 4.8 \\
Zhong Jiang, Sichuan Province, China & 0.13 & 0.16 & 4.4 \\
Lu Shi, Henan Province, China & 0.17 & 0.22 & 5.6 \\
Ping Ding Shan, Henan Province, China & 0.22 & 0.41 & 5.4 \\
Luo Yang, Henan Province, China & 0.17 & 0.33 & 6.7 \\
Shang Luo, Shaanxi Province, China & 0.18 & 0.25 & 5.6 \\
Mean & 0.16 & 0.27 & 5.4 \\
S.D. & 0.045 & 0.084 & 0.73 \\
\hline & & &
\end{tabular}

units. The measurement uncertainties for the HPLC determination of tanshinone IIA, rosmarinic acid, and salvianolic acid B are summarized in Table 3 .

HPLC Quantitative Analysis Results Seven batches of Radix Salviae Miltiorrhizae samples collected from representative regions of China and three batches of processed medicinal materials purchased from a Hong Kong market were quantitatively determined using the reverse-phase HPLC methods developed in this paper. Each sample was analyzed in duplicate to determine the mean contents of three pharmacologically active marker compounds in crude drugs (Table 4).

The quantitative results showed that the established assay method is suitable for the evaluation of the quality not only of the crude drug but also of the processed medicinal materials. Table 5 shows the contents of marker compounds in three batches of the processed Radix Salviae Miltiorrhizae purchased. The contents of three pharmacologically active compounds in the processed materials were much lower than those in the crude drugs. Thus studies of quality standards of Chinese herbal medicines should be based on the representative crude drug samples. The content limits of marker compounds in crude drugs can be proposed according to the determination results of representative crude drug samples and MU. 
Table 5. Contents of Three Marker Compounds in Processed Medicinal Materials of Radix Salviae Miltiorrhizae Purchased from Three Companies in Hong Kong

\begin{tabular}{cccc}
\hline \hline Source & $\begin{array}{c}\text { Tanshinone } \\
\text { IIA (\%) }\end{array}$ & $\begin{array}{c}\text { Rosmarinic } \\
\text { acid (\%) }\end{array}$ & $\begin{array}{c}\text { Salvianolic } \\
\text { acid B (\%) }\end{array}$ \\
\hline Company A & 0.047 & 0.077 & 2.2 \\
Company B & 0.12 & 0.10 & 3.2 \\
Company C & 0.064 & 0.062 & 2.2 \\
Mean & 0.077 & 0.080 & 2.5 \\
S.D. & 0.038 & 0.021 & 0.59 \\
\hline
\end{tabular}

In conclusion, a comprehensive quality assessment method for Radix Salviae Miltiorrhizae, was established in this paper. Compared with the previous quality standard of Radix Salviae Miltiorrhizae, ${ }^{1)}$ our improved methods include HTPLC fingerprints of both water-soluble and nonpolar compounds and HPLC fingerprint of total extract containing a large amount of chemical information that can be used for the identification of the crude drug, and HPLC determination of three pharmacologically active marker compounds directly associated with the quality of herbal medicines. The method of HPTLC fingerprinting identification has the advantages of simplexity, rapidity, and visuality, while the HPLC fingerprint method has the advantages of specificity, powerful separation ability, and increased chemical information. These two fingerprint methods can improve the reliability of identification of Chinese herbal medicines. In the HPLC fingerprinting procedure, the RRT of characteristic peaks was found to be an ideal quantitative parameter for the authentication of crude drugs if characteristic peaks and marker compounds are carefully selected. The MU evaluated from the HPLC validation data will be considered as an important parameter for proposing the content limits of respective marker compounds in the quality standard of medicinal herbs.

\section{Experimental}

Herbal Materials Reference Chinese Materia Medica powder of Radix Salvia Miltiorrhizae was purchased from the National Institute for the Control of Pharmaceutical and Biological Products (Beijing, China). Seven samples of crude drug were collected from the cultivation base of Salvia miltiorrhiza in different regions of China and were air-dried according to the procedure described in the Chinese Pharmacopoeia (2000 edition). Three samples of processed medicinal materials (after washing with water, the crude drugs were softened and sliced) were purchased from three Chinese herbal medicine companies in Hong Kong. All of them were identified by one of the authors (Z. Zhao) and deposited in the Centre of Chinese Materia Medica, Hong Kong Baptist University.

Reference Standards Cryptotanshinone, danshensu, tanshinone IIA, and salvianolic acid B (purity $>98 \%$ ) were purchased from the National Institute for the Control of Pharmaceutical and Biological Products (Beijing, China). Rosmarinic acid (purity $>97 \%$ ) was obtained from Sigma (St. Louis, MO, U.S.A.). Lithospermic acid (purity $>96 \%$ ) was isolated in our laboratory and the identity and purity were confirmed by chromatographic methods (TLC and HPLC) and by comparison with the published spectral data.

Reagents Acetonitrile and methanol of HPLC grade and chloroform, cyclohexane, dichloromethane, diethyl ether, ethanol, ethyl acetate, petroleum ether $\left(60-80^{\circ} \mathrm{C}\right)$, and toluene of analytical grade were purchased from Labscan (Stillorgan, Ireland). Formic acid of GR grade for HPLC analysis was obtained from Merck (Darmstadt, Germany). Water for HPLC analysis was prepared using a Milli-Q water purification system (Millipore Corp., Bedford, MA, U.S.A.).

Sample Preparation for HPTLC Identification One gram of powdered sample was extracted with $10 \mathrm{ml}$ of diethyl ether for $1 \mathrm{~h}$ at room temperature with occasional shaking. After centrifugation, the supernatant was quickly dried in a water bath. The residue was then dissolved in $0.5 \mathrm{ml}$ of methanol as the TLC test solution for the nonpolar compounds.

Another $1 \mathrm{~g}$ of powdered sample was decocted for $30 \mathrm{~min}$ with $50 \mathrm{ml}$ of water. After centrifugation, the supernatant was adjusted to $\mathrm{pH} 2$ with $\mathrm{HCl}$ $2 \mathrm{M}$ with a $\mathrm{pH}$ meter. The solution was then extracted with ethyl acetate $(3 \times 10 \mathrm{ml})$ after filtration. The combined organic layer was concentrated to dryness under a vacuum. The residue was dissolved in $2 \mathrm{ml}$ of ethyl acetate as TLC test solution for the water-soluble compounds.

HPTLC Procedure for Identification HPTLC silica gel 60 F254 plates $(10 \mathrm{~cm} \times 10 \mathrm{~cm})$ were Merck products. The plates were dried for $30 \mathrm{~min}$ at $105^{\circ} \mathrm{C}$ before use. Test solutions $(2 \mu \mathrm{l})$ of the reagent blank, sample, sample duplicate, reference standard, spiked sample ( $1 \mu 1$ of sample $+1 \mu 1$ of standard) and reference Chinese Materia Medica were applied on the plates with an TLC system (Camag, Muttenz, Switzerland). The plates were then developed in a double-trough $10 \times 10$-cm chamber with a stainless steel lid which was preequilibrated with the mobile phase. For HPTLC separation of nonpolar compounds, petroleum ether-ethyl acetate-cyclohexane $(5: 3: 2)$ was used as the developing solvent. After developing over a path of $4.5 \mathrm{~cm}$, the plate was dried in air and imaged under UV light $(254 \mathrm{~nm})$ and visible light. For HPTLC separation of water-soluble compounds, chloroform-ethyl acetate-toluene-formic acid-methanol $(15: 20: 10: 10: 1)$ was chosen as the solvent system. After developing over a path of $7 \mathrm{~cm}$, the plate was dried in air and imaged under UV light $(254 \mathrm{~nm})$. The plate was then sprayed with $2 \% \mathrm{FeCl}_{3} / \mathrm{EtOH}$, heated at $110^{\circ} \mathrm{C}$ for $10 \mathrm{~min}$ and was imaged immediately under visible light.

Sample Preparation for HPLC Fingerprinting The powdered crude drug $(0.2 \mathrm{~g})$ was extracted with $5 \mathrm{ml}$ of methanol for $30 \mathrm{~min}$ by sonication. After centrifugation, the supernatant was transferred into a $25-\mathrm{ml}$ amber volumetric flask. The sample was reextracted twice with $5 \mathrm{ml}$ of methanol and the supernatants were combined. The residue was then transferred into a 50$\mathrm{ml}$ round-bottomed flask and refluxed with $10 \mathrm{ml}$ of water for $30 \mathrm{~min}$. The suspension was centrifuged at $5000 \mathrm{rpm}$ for $10 \mathrm{~min}$, and the supernatant was combined with the above methanol solution which was made up to $25 \mathrm{ml}$ with water. The sample solution was filtered through a $0.22-\mu \mathrm{m}$ nylon filter (Iwaki, Japan) into a HPLC amber sample vial for HPLC fingerprinting analysis.

HPLC Conditions for HPLC Fingerprinting An HP 1100 system (Hewlett-Packard, Wilmington, DE, U.S.A.) consisting of a G1312A binary pump, G1329A automatic sample injector, and G1315A diode array detector was used to perform HPLC analysis. The HPLC fingerprint was carried out on a C18 column (Alltech, Alltima C18, $4.6 \mathrm{~mm} \times 250 \mathrm{~mm}, 5 \mu \mathrm{m}$ ) at ambient temperature with a sample injection volume of $20 \mu \mathrm{l}$. The mobile phase was a gradient elution of water-acetonitrile-formic acid $(90: 10: 0.4, \mathrm{v} / \mathrm{v})$ and acetonitrile (B), commencing with $0 \% \mathrm{~B}$, rising to $30 \% \mathrm{~B}$ after $40 \mathrm{~min}$, then to $80 \% \mathrm{~B}$ after $50 \mathrm{~min}$, and $85 \% \mathrm{~B}$ after $70 \mathrm{~min}$ at a flow rate of $1.0 \mathrm{ml} / \mathrm{min}$. The fingerprint profiles were recorded at an optimized wavelength of $280 \mathrm{~nm}$.

Identification of Peaks in HPLC Fingerprints The peaks in HPLC fingerprinting were identified by comparing the retention times of danshensu, rosmarinic acid, lithospermic acid, salvianolic acid B, cryptotanshinone, and tanshinone IIA in chromatograms of the extracts with those of the authentic reference standards and using LC-MS-MS.

Preparation of Standard Solutions for HPLC Quantitation Stock solution of standard tanshinone IIA was obtained by dissolving the crystal in least amount of dichloromethane and adding methanol to obtain a concentration of $500 \mathrm{mg} / \mathrm{l}$. Stock solutions of rosmarinic acid and salvianolic acid B were prepared by dissolving each compound in methanol-water $(50: 50)$ to reach a concentration of $2000 \mathrm{mg} / \mathrm{l}$. The solutions were then stored at $-10{ }^{\circ} \mathrm{C}$ in the dark.

The concentrations of reference standard tanshinone IIA used for calibration were $5.00,10.0,20.0,50.0$, and $100 \mathrm{mg} / 1$ in methanol. The concentrations of standard solutions of polyphenolics used for calibration were obtained by diluting the stock solutions with methanol-water $(50: 50, \mathrm{v} / \mathrm{v})$ to $1.00,2.00,5.00,10.0$, and $20.0 \mathrm{mg} / 1$ for rosmarinic acid and 20.0, 40.0, 100, 200 , and $400 \mathrm{mg} / 1$ for salvianolic acid B.

Sample Preparation for HPLC Quantitation About $0.50 \mathrm{~g}$ of powdered crude drug was accurately weighed into a 10-ml centrifugal tube. A 5$\mathrm{ml}$ portion of methanol-dichloromethane $(8: 2)$ was added, and the suspension was sonicated for $30 \mathrm{~min}$. After centrifugation, the supernatant was carefully transferred into a $25-\mathrm{ml}$ volumetric flask. The sample was then reextracted with $5 \mathrm{ml}$ of methanol-dichloromethane four times and the supernatants were combined into the volumetric flask which was made up to $25 \mathrm{ml}$ with methanol-dichloromethane $(8: 2)$. The sample solution was filtered through a $0.45-\mu \mathrm{m}$ PTFE filter (Iwaki, Japan) as the test solution for 
quantitative analysis of tanshinone IIA.

About $0.10 \mathrm{~g}$ of powdered crude sample was accurately weighed into a 10 - $\mathrm{ml}$ centrifugal tube. Methanol-water $(6: 4) 5 \mathrm{ml}$ was added. The suspension was sonicated for $30 \mathrm{~min}$. After centrifugation, the supernatant was carefully transferred into a $25-\mathrm{ml}$ volumetric flask. The sample was then reextracted with $5 \mathrm{ml}$ of methanol-water four times and the supernatants were combined into the volumetric flask which was made up to $25 \mathrm{ml}$ with methanol-water $(6: 4)$. The sample solution was filtered through a $0.22-\mu \mathrm{m}$ nylon filter as the test solution for quantitative analyses of salvianolic acid B and rosmarinic acid.

HPLC Conditions for Quantitation For quantitation of tanshinones, a C18 column (Alltech, Alltima C18, $4.6 \mathrm{~mm} \times 150 \mathrm{~mm}, 5 \mu \mathrm{m}$ ) was used. Solvent of methanol/water $(75: 25)$ and detection wavelength of $270 \mathrm{~nm}$ were selected for the assay. The flow rate was $1 \mathrm{ml} / \mathrm{min}$, and the injection volume was $20 \mu 1$.

For quantification of polyphenolics, the separation was performed on a C18 column (Alltech, Alltima C18, $4.6 \mathrm{~mm} \times 250 \mathrm{~mm}, 5 \mu \mathrm{m}$ ). The mobile phase was water-acetonitrile-formic acid $(22: 78: 0.4)$. The analysis was carried out under isocratic conditions at a flow rate of $1 \mathrm{ml} / \mathrm{min}$, and $330 \mathrm{~nm}$ was the wavelength for detection. The injection volume was $20 \mu \mathrm{l}$.

Validation of Quantitative Methods Five-point calibration curves were based on linear regression analysis. The intraday precisions of injection were calculated with the results obtained by analyzing the middle point standard solutions of the calibration curve five times. Five replicate analyses of one batch of Radix Salviae Miltiorrhizae sample were carried out to evaluate the repeatability of the quantitative procedure. The recovery studies were conducted by spiking the same batch of Radix Salviae Miltiorrhizae sample five times in the repeatability study of tanshinone IIA, rosmarinic acid, and savianolic acid B standards at $c a$. $0.4,1$, and $10 \mathrm{mg} / \mathrm{g}$, respectively. Limits of detection of tanshinone IIA, rosamrinic acid, and salvianolic acid B were based on visual evaluation (signal-to-noise ratio of $3: 1$ ) by analyzing the diluted sample extract with known concentrations of analyte. The LOQ of tanshinone IIA, rosmarinic acid, and salvianolic acid B in Radix Salviae Miltiorrhizae were primary estimated by the signal-to-noise ratio (about $10: 1$ ). Then five replicate analyses of blank matrix (Radix Ginseng) spiked with estimated amounts of tanshinone IIA, rosmarinic acid, and salvianolic acid B were performed. The spiked amount was $c a .0 .04 \mathrm{mg} / \mathrm{g}$ of the standards of tanshinones, $0.1 \mathrm{mg} / \mathrm{g}$ of rosmarinic acid, and $0.5 \mathrm{mg} / \mathrm{g}$ of salvianolic acid B, respectively. The recoveries of the spiking tests fell within the range of 90 $110 \%$, and the RSDs were less than $10 \%$.

Acknowledgments This work is part of a project on the study of some common Chinese Materia Medica in Hong Kong, commissioned by Department of Health, Hong Kong Government of Special Administrative Region, PR China. Part of the financial support was also provided by the Key Tech- nologies Research and Development Programme of the Tenth Five-year Plan of the Ministry of Science and Technology of China (2002BA906A29-3, 2003-2004), National Basic Research Priorities Programme of the Ministry of Science and Technology of China (2002DEA20021-3, 2003-2004), and Scientific Research Plan Programme of the Shanghai Science and Technology Committee of China (03DZ19548).

\section{References}

1) Pharmacopoeia Commission of People's Republic of China, "Pharmacopoeia of the People's Republic of China," 2000 ed., Chemical Industry Press, Beijing, China, 2000, pp. 57-58.

2) Guo J. X., "Research and Clinical Application of the Root of Salvia Miltiorrhiza Bunge," Chinese Medicine and Technology Press, Beijing, China, 1992.

3) Editorial Board of China Herbal, State Administration of Chinese Traditional Medicine, "China Herbal," Vol. 7, ed. by Ying X. X., Yu H. A., Shanghai Medicine and Technology Press, Shanghai, China, 1999, pp. $169-186$

4) Cui L., Wu T., Liu Y. Y., Acta Pharm. Sin., 25, 678-684 (2004).

5) Liang Y., Yang Y. M., Yuan S. L., Chin. Trad. Herb. Drugs, 31, 304306 (2000).

6) Lu Y. R., Foo L. Y., Phytochemistry, 59, 117-140 (2002).

7) Zhou C. X., Luo H. W., Niwa M., J. Chin. Pharm. Univ., 30, 411-416 (1999).

8) Li L. N., J. Chin. Pharm. Sci., 6, 57-64 (1997).

9) Lay I. S., Chiu J. H., Shiao M. S., Planta Med., 69, 26-32 (2003).

10) Luo G. A., Wang Y. M., Chin. Trad. Pat. Med., 19, $44-47$ (1997).

11) State Drug Administration of China, Chin. Trad. Pat. Med., 22, 671675 (2000).

12) Schaneberg B. T., Crocketts S., Bedir E., Khan I. A., Phytochemistry, 62, 911-918 (2003).

13) Cai Z. W., Lee F. S. C., Wang X. R., J. Mass Spectrometry, 37, 10131024 (2002).

14) Joseph S., J. AOAC Int., 86, 873-881 (2003).

15) Bernard F., Joseph S., "Practical Thin-Layer Chromatography," CRC Press Inc., Boca Raton, Florida, 1996.

16) Luo G. A., Hu P., Jiang Z.-H., Modernization of Traditional Chinese Medicine and Materia Medica, 6, 10-15 (2004).

17) Eurachem/Citac Guide, "Quantifying Uncertainty in Analytical Measurement," 2nd ed., ed. by Ellison S. L. R., Rosslein M., Williams A., www.eurachem.org, 2000.

18) VAM Project 3.2.1, "Development and Harmonisation of Measurement Uncertainty Principles," ed. by Barwick V. J., Ellison S. L. R., LGC press, Teddington, UK, 2000. 